136 WHICH BLOCKS CAN YOU DO? AN ASSESSMENT OF ANAESTHETIC TRAINEE CONFIDENCE PERFORMING COMMON PERIPHERAL NERVE BLOCKS

D Luff*, F Moosa, N Sadavarte, N Pinnamaneni, N Bedforth. Nottingham University Hospitals Trust, Nottingham, UK

\subsection{6/rapm-2021-ESRA.136}

Background and Aims Recent opinion has suggested focusing training on a small number of 'Plan A blocks' to improve basic regional anaesthesia competence amongst non-regional enthusiasts ${ }^{1}$. We assessed trainee confidence performing Plan A blocks with indirect supervision. We also sort trainee opinion regarding which techniques should be designated as essential competencies and how our regional anaesthesia training could be improved.

Methods We performed an email survey of all anaesthetic trainees within the East Midlands School of Anaesthesia exploring their confidence in performing regional anaesthesia.

Results We received 40 trainee responses (7 CT1-CT2, 13 ST3-ST5, 20 ST6-ST7). Trainees confident in performing plan A blocks under indirect supervision were (numbers are expressed as $\%$ [positive responses/total responding]): Interscalene 60 [24/40]; axillary 60 [24/40]; [NB1] femoral 87.5 [35/ 40]; adductor canal 42.5 [17/40]; popliteal 65 [26[NB2]/40]; erector spinae 10 [4/40]; rectus sheath blocks 42.5 [17/40]. Trainees responding that individual blocks should be included as core competencies: Interscalene 87.2 [34/40]; axillary 84.6 [33/40]; femoral 87.5 [35/40]; adductor canal 56.4 [22/40]; popliteal 76.9 [30/40]; erector spinae 10 [4/40]; rectus sheath blocks $67.5[27 / 40] .80 \%$ of our trainees expressed a desire

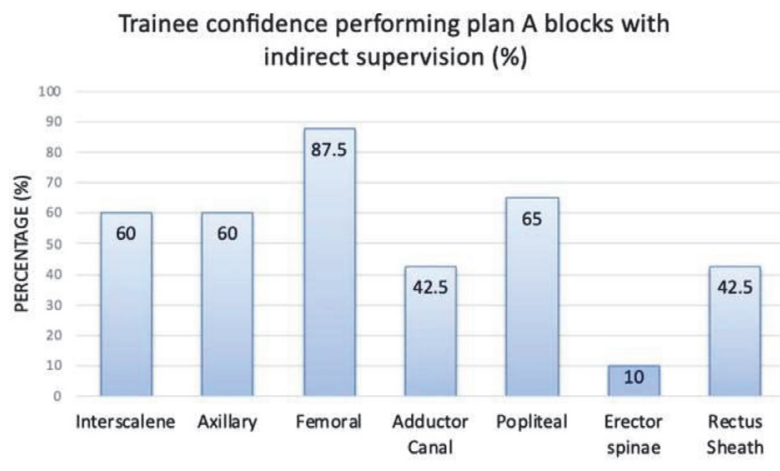

Abstract 136 Figure 1

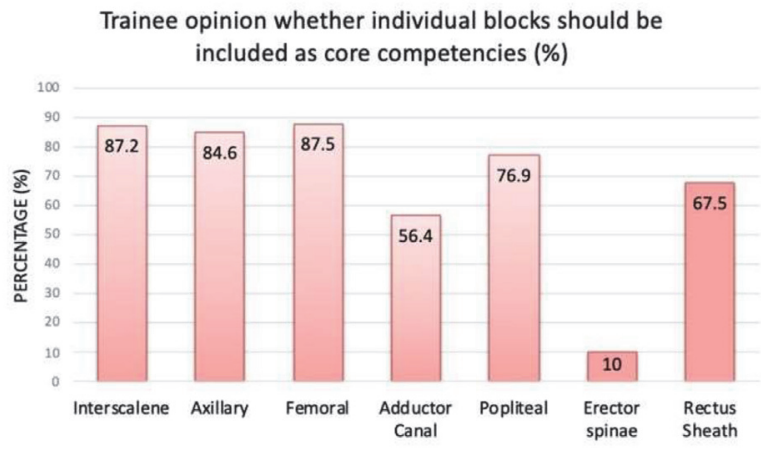

Abstract 136 Figure 2 for regular local teaching and 50\% wanted teaching to focus on a reduced number of essential blocks.

Conclusions The majority of our trainees felt confident performing most plan A nerve blocks with indirect supervision. Opinion regarding which blocks should be essential competencies mirrored these results. We have commenced regular small group teaching sessions focusing on Plan A blocks.

\section{AVOIDING INTERNAL THORACIC ARTERY INJURY DURING PRE-INCISION TRANSVERSUS THORACIC MUSCLE PLANE BLOCK IN CORONARY ARTERY BYPASS GRAFT SURGERY: A TECHNICAL REPORT}

AAGPS Jaya*, AR Tantri, RB Sukmono. Universitas Indonesia/Dr. Cipto Mangunkusumo Hospital, Department of Anesthesiology and Intensive Care, Faculty of Medicine, Jakarta Pusat, Indonesia

\subsection{6/rapm-2021-ESRA.137}

Background and Aims Internal thoracic artery (ITA) is the gold-standard conduit in coronary artery bypass graft surgery (CABG). Therefore, ITA injury should be avoided during the pre-incision transversus thoracic muscle plane block (TTPB) in CABG. Hence, some anesthesiologists prefer to perform TTPB postoperatively. However, pre-incisional TTPB has potential advantages in reducing the requirement for intraoperative opioids and decreasing pain sensitization. We describe a sequence of steps in performing pre-incision TTPB to avoid ITA injury.

Methods We performed a pre-block scan by placing a high-frequency linear ultrasound probe transversely at desired intercostal space (ICS), applied color Doppler to identify ITA (figure 1 ), and marked the skin above ITA. After aseptic skin preparation, we put the ultrasound probe in sagittal orientation on the skin mark and then moved laterally until the lateral edge of transversus thoracic muscle (TTM) is visible. We target the
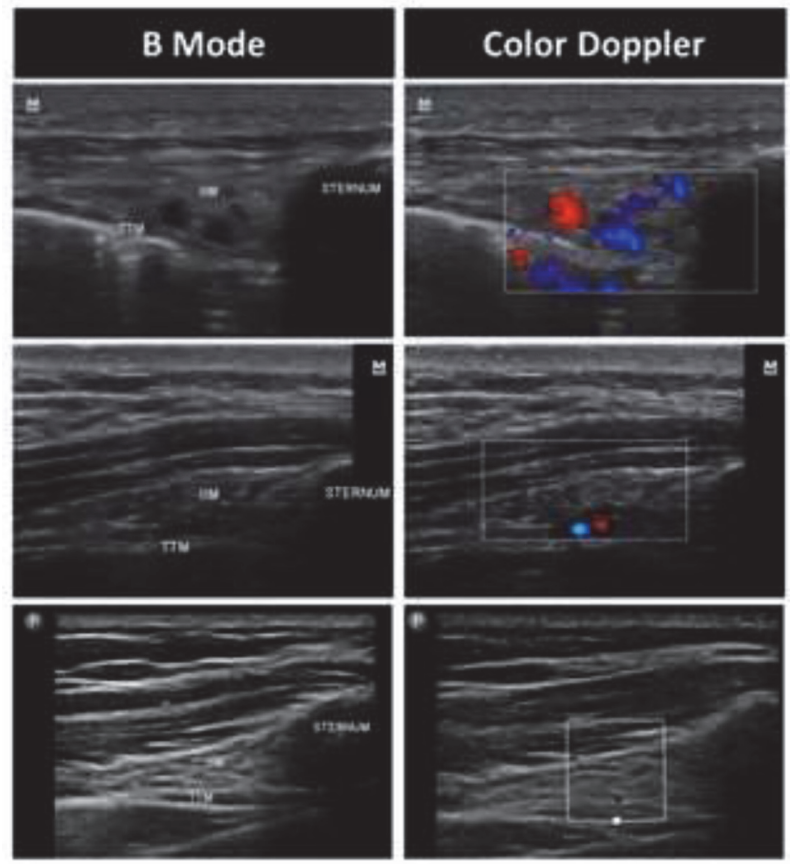

Abstract 137 Figure 1 

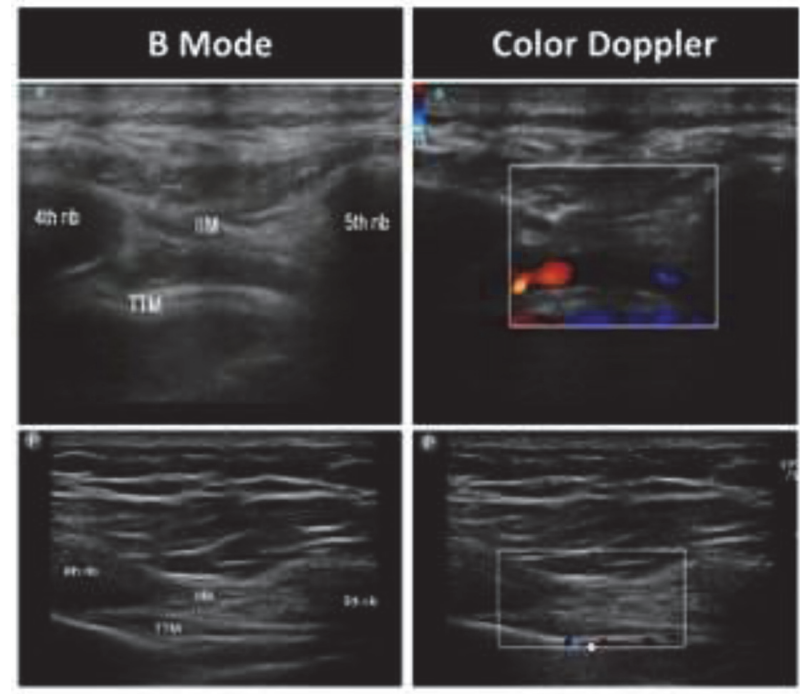

Abstract 137 Figure 2

plane between internal intercostal muscle and TTM, lateral to ITA.

Results TTPB can be performed at the $3^{\text {rd }}$ or $4^{\text {th }}$ ICS, using a transverse or sagittal approach. A sagittal approach at the $4^{\text {th }}$ ICS is recommended due to the wider LA spread. We may encounter several challenges during TTPB performance: ITA location is in the same plane as the target of TTPB, ITA varies in size, identification of ITA is not always easy, and ITA might mimic TTM (figure 2), which prone to accidental puncture.

Conclusions We have developed a sequence of steps that may guide anesthesiologists to perform the safe and effective TTPB, particularly when the block is performed before the incision in CABG.

\section{COMPARISON OF ANTERIOR, POSTERIOR AND LATERAL APPROACHES OF ULTRASOUND GUIDED QUADRATUS LUMBORUM BLOCK IN PEDIATRIC PATIENTS UNDERGOING LOWER ABDOMINAL SURGERY: A PROSPECTIVE RANDOMIZED CONTROL TRIAL}

AS K*, A Kumar, B Kumar, C Sinha, A Kumar, P Kumari. All India Institute of Medical Sciences, Patna, Patna, India

\subsection{6/rapm-2021-ESRA.138}

Background and Aims Background: Changing concepts have made pediatric pain management providers think beyond traditional opioid management to help alleviate pediatric pain ${ }^{1}$. Ultrasound-guided quadratus lumborum (QL) block is now considered as one of the novel abdominal block ${ }^{2}$.

Aims: To compare the post-operative analgesic effect between the three approaches of USG guided quadratus lumborum block (QLB).

Methods It's a prospective, double-blinded, randomized trial of pediatric patients. 30 were recruited and randomized into 3 groups,

Group A, Trans-muscular/anterior QLB, Group B, Lateral QLB, Group C, Posterior QLB

After general anaesthesia, QLB was given with $0.2 \%$ ropivacaine, $0.5 \mathrm{ml} / \mathrm{kg}$. Primary outcome - total fentanyl consumption in the first 24 hours of post-operative period. Pain in post-operative period was accessed using FLACC score $^{3}$.

Results All baseline demographics were comparable. Total fentanyl consumption in the first 24 hours of post-operative

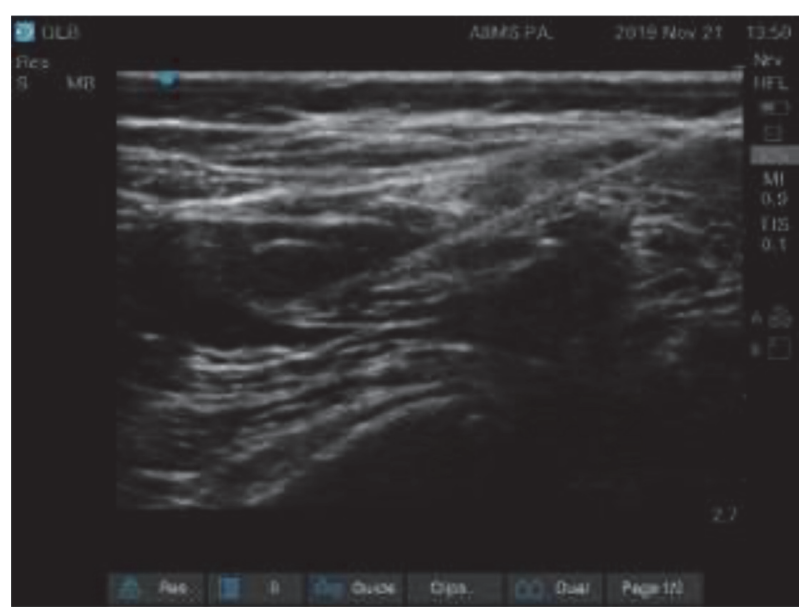

Abstract 138 Figure 1

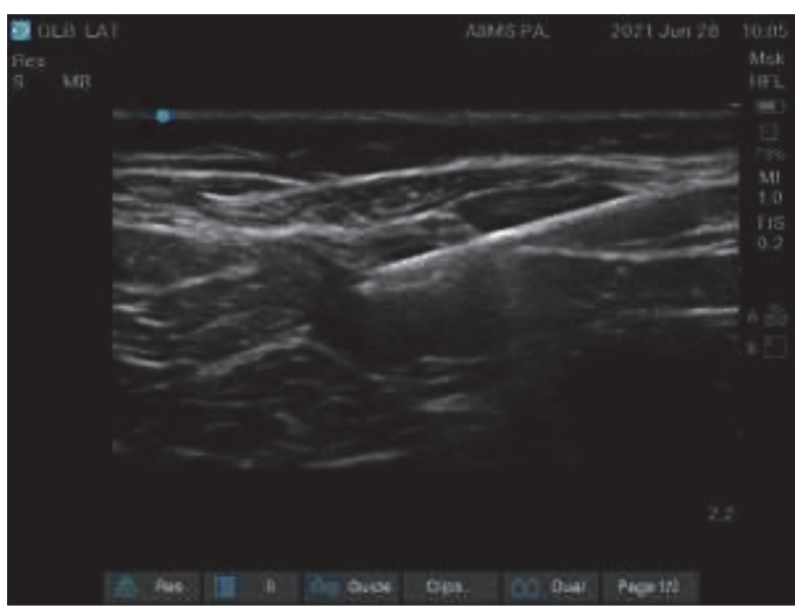

Abstract 138 Figure 2

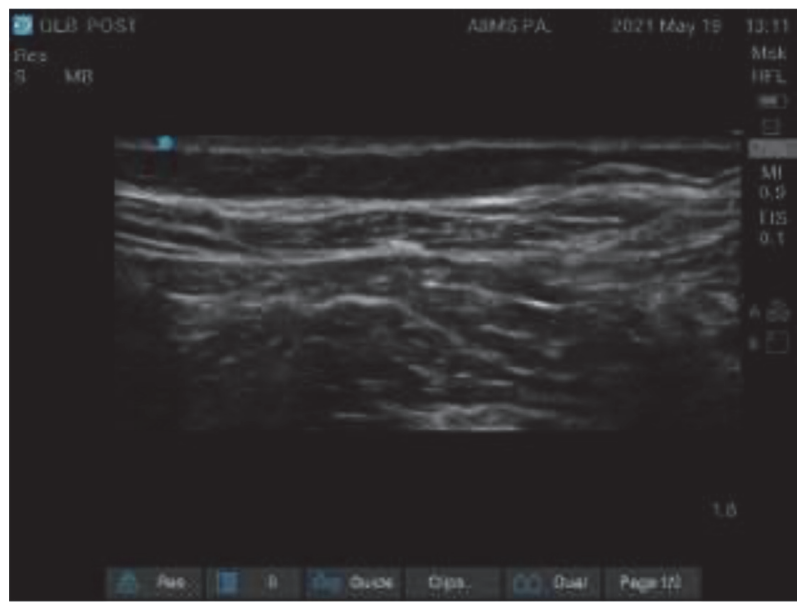

Abstract 138 Figure 3 